\title{
The Application of a New Type of Catheter-Specific Pants for Patients with Indwelling Catheter After Transurethral Prostate Resection
}

\author{
Xiaoxia Guo ${ }^{1}$, Qingran Lin ${ }^{2, *}$ \\ ${ }^{1}$ Department of Urology, The First Affiliated Hospital of Jinan University, Guangzhou, China \\ ${ }^{2}$ Department of Urology, The Third Affiliated Hospital of Southern Medical University, Guangzhou, China \\ Email address: \\ qingranlin@yeah.net (Qingran Lin) \\ *Corresponding author
}

\section{To cite this article:}

Xiaoxia Guo, Qingran Lin. The Application of a New Type of Catheter-Specific Pants for Patients with Indwelling Catheter After Transurethral Prostate Resection. American Journal of Nursing Science. Vol. 6, No. 3, 2017, pp. 271-273. doi: 10.11648/j.ajns.20170603.25

Received: March 28, 2017; Accepted: April 7, 2017; Published: May 25, 2017

\begin{abstract}
To explore the new catheter-special pants design and the application for patients with indwelling catheter after transurethral prostate resection, 156 patients after transurethral prostate resection were divided into two groups randomly: experimental group wearing newly designed pants and control group wearing normal pants after transurethral prostate resection; to compare the state of restlessness and the times patients tearing the catheter secondary fixation tape and pulled out personally unplanned. Experimental group wearing newly designed pants showed only one case of catheter pulled out, none of fixation tape was teared, while in the control group, 9 cases of catheter were pulled out personally, 21 cases of tape teared, causing some degrees of urethral injury, delayed the retention time of catheter. There were significant differences between the two groups. The application of newly designed pants shielding the catheter decreases the incidence of catheter pullout after transurethral prostate resection surgery, avoids urethral injury and increased the retention time, improves the functional recovery with no increased cost, which is worth promoting the application in ordinary clinical nursing.
\end{abstract}

Keywords: Newly Designed Pants, Postoperative Indwelling Catheter, Transurethral Prostate Resection

\section{Introduction}

Prostatic hyperplasia is a common disease in the elderly, and transurethral prostate resection is the golden procedure for the treatment of prostatic hyperplasia [1-3]. Indwelling catheter is conventionally applied to tract and oppress the prostate fossa for hemostasis, drainage of the urine and to prevent bladder neck stenosis [4, 5]. According to the literature and in our daily nursing work, patients awaken after the transurethral prostate resection show expressions of agitation and noisy, forced out of bed and trying to plug all the kind of tubes reached, such as oxygen tubes, infusion tube and catheter $[6,7]$. In order to decrease the chance for patients to pullout the catheter personally for the smooth postoperative recovery, we designed a new kind of pants which can cover the urine, to grantee the fixation of catheter during agitation stage.

\section{Clinical Data and Methods}

\subsection{Clinical Data}

156 case under transurethral prostate resection in the Affiliated Hospital of Jinan University from Jan 2015 to Dec 2015 were collected into the study. 76 cases from Jan 2015 to Jun 2015 were included as control group and 80 cases from Jul 2015 to Dec 2015 were included as experimental group; aging from 55-92 years old, 71.8 of average in control group and 72.1 of average in the experimental group, no significant difference between the two groups $(\mathrm{P}>0.05)$.

\subsection{Methods}

20\# three chamber silicone catheters were applied to both groups, urine tubes were injected with $30 \mathrm{ml}$ of liquid for the 
fixation and all the other handling methods were both stayed the same in both the two groups.

\subsubsection{Special Pants Designing}

In the control group, the catheters were applied and fixed as normal method. The experimental group used the newly designed pants as follows: the new type of pants have special hole for the urine tube, diaper hanging lace, urine bag pocket, cover cloth and fixed buckle for adjusting the length; (1) using the cotton patients pants, making the changes in the left leg. Open a $10 \mathrm{~cm}$ hole $50 \mathrm{~cm}$ distant from the belts; (2) making a cloth pocket $48 \mathrm{~cm}$ distant from the belts; (3) making a cover of $5 \mathrm{~cm}$ in the left leg, with the bottom unfixed; (4) both the legs, making buckles 10 and $15 \mathrm{~cm}$ from the bottom (Figure 1).

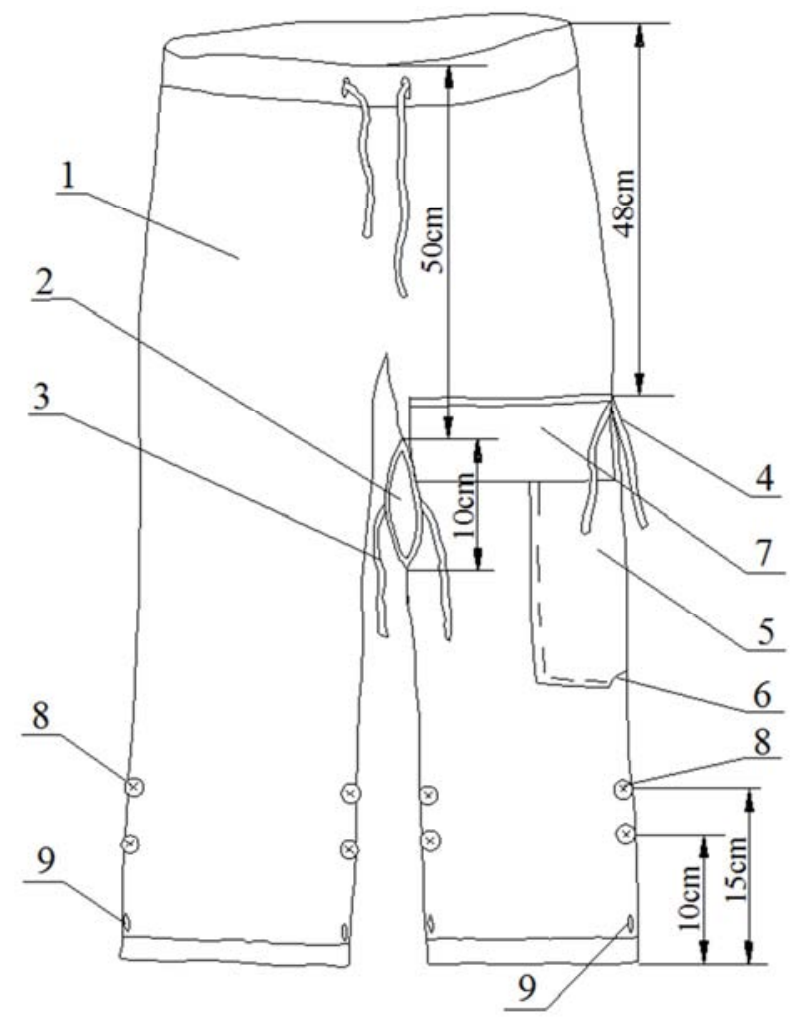

Figure 1. The newly designed pants.

1: cotton pants as normal; 2 : outlet for the urinary catheter; 3 : lace for inner-side hole; 4: diaper hanging lace; 5: bag for the urine bag; 6 : curved opening; 7: cloth cover; 8 : buckles; 9: buttonhole.

\subsubsection{The Use of Newly Designed Pants}

Immediate wear the newly designed pants after the surgery, fix the outreach of the tube within the thigh medial with "high-hold the platform method". The tube is parallel to the thigh, urethral trigeminal transduction go through the outlet (Figure 1: position 2). When patients leave the bed, the urinal bag is placed in the bag (Figure 1: position 5), redundant tube is fixed with the lace (Figure 1: 4 position) and cover with the cotton (Figure 1: position 7). The length of the pants can be adjusted by using the buckles (Figure 1: position 8 and 9). Families should be accompanied for patients within agitation period and no tools can be used for the activity restriction.

\subsubsection{Statistical Analysis}

All statistical analyses were performed using the Statistical Package for the Social Sciences, version 20.0 for Windows. A two-sided P-value $<0.05$ was considered statistically significant.

\section{Results}

Agitation level evaluation: (1) level 0: quiet and cooperative; (2) level 1: light limb agitation, can be comfort by talking; (3) level 2: agitated by no stimulation, trying to resist and to remove the pipe; (4) level 4: incentive struggling, needs more people to care. Level 1 to 4 is evaluated with agitation. The agitation cases in the two groups were listed in Table 1 . The cases of catheter fixation and plugged personally were listed in Table 2. The results showed that by the use of newly designed pants, the agitation of patients decreased and patients in level 0 increased, indicating that the patients felt more comfortable when using the newly designed pants (Table 1). Also, the cases of tearing the fixed tape or plugging the tube personally decreased, indicating that patients were willing to remain the catheter (Table 2). In all, the data suggest the successful application of the newly designed pants.

Table 1. Agitation performance between the two groups.

\begin{tabular}{llllll}
\hline Groups & N & Level 0 & Level 1 & Level 2 & Level 3 \\
\hline Experimental & 80 & 43 & 28 & 8 & 1 \\
Control & 76 & 14 & 32 & 21 & 9 \\
\hline
\end{tabular}

Table 2. Cased of fixation teared and tube plugged personally in the two groups.

\begin{tabular}{llll}
\hline Groups & N & Fixed tape teared & Tube plugged personally \\
\hline Experimental & 80 & 0 & 1 \\
Control & 76 & 21 & 9 \\
\hline
\end{tabular}

\section{Discussion}

Patients in the agitated recovery period show excitement and orientation disorder, performing limb unconscious movements, irrational words, crying or moaning and delusion thoughts, which are all belong to the complications of recovery period $[8,9]$. Urine tubes are often needed to be removed for obvious agitated patients to relief the pain. Postoperative indwelling catheter is the critical step for transurethral prostate resection surgery. The application of newly designed pants which cover the tubes, protected the patients privacy, decreased unplanned plug, functioned for the recovery for patients.

\subsection{Firm Fixed Catheter Tubes}

Normal pants have no special outlet for the catheter, the tubes often go out from the trousers and fixed on the leg $5 \mathrm{~cm}$ below the position of groin, which is applied in control group. In this way, the hands of patients would touch the tube and fixed tape, making the tubes randomly pulled, tape teared or even the tube pulled out. In the control group, there were 21 cases of fixed tape teared twice, 9 cased of catheter tubes 
pulled out and 1 case of tube plugged twice $48 \mathrm{~h}$ postoperative. In this case, there were a large number of urethral bleeding, cannot urinate making the blood pressure drop, and was sent to ICU for drug comfort for 5 days and carry catheter for 2 weeks. In the experimental group, with special designed outlet for the tube and fixed and hide tubes distant from the hands, largely deceased the risks in the control group. In the experimental group, there is an Alzheimer's patients, due to the humpback he cannot be at supine/sitting position, making the patients severely agitated and pulled out the tube. Because of the mental behavior of AD patients, there people show high risk of pulling out the tube personally, which are the focus of the study [10-12].

\subsection{The Drainage Compliance}

In the control group, the tube folds back to the pubic then out the body, with a U turn for the fluid to flow out, making difficulties for the urine outflow. However, in the experimental group, the tube is parallel to the urethra, making the urine flow out smooth, without backflow and retention, preventing the infection. Drainage not smooth is the cause of bladder spasm. Accordingly, tube stimulation and the pain composed the agitation of catheter carrying. Compared to the control group, the cased of agitated patients were largely deceased by the application of the newly designed pants. Thus, with the new pants, the urine is flowed out fluently, efficiently decreased the pressure of bladder and decreased the agitation.

\subsection{Privacy Protection}

After the surgery of transurethral prostate resection, there is often small blood clots or tissue fragment which would block the catheter. When washing the tubes, patients in the control group should take of the trousers and explore perineum and this is especially embarrassing for female patients. However, in the experimental group, patients only need to lose the lack, which is largely protected the privacy.

\subsection{Preventing Fall Injury}

Adult pants in the hospital have the same size in the pant legs, though varies in size. In the study, the subjects were all the elderly, making the pant legs are often longer than patients' legs, leading to the easily fall injury [13, 14]. However, in our newly designed pants, the lengths of the legs can be adjusted accordingly, which can largely decrease the risk of fall injury.

In summary, the application of newly designed pants for transurethral prostate resection can significantly decrease the rate of catheter pulled out personally and unplanned, decrease the agitation, increase the comfort and increase the safety for off-bed activity, thus promoting the recovery, which brings large economic and social benefits, worthy of clinical promotion.

\section{References}

[1] Wendt-Nordahl, G., Y. Cao, A. Hacker, M. S. Michel, and T. Knoll, Transurethral resection of the prostate: defending its leading position in the management of benign prostatic enlargement. Minerva Urol Nefrol, 2009, 61 (3): 291-300.

[2] Hawary, A., K. Mukhtar, A. Sinclair, and I. Pearce, Transurethral resection of the prostate syndrome: almost gone but not forgotten. J Endourol, 2009, 23 (12): 2013-20.

[3] Lumen, N., P. Hoebeke, P. Willemsen, B. De Troyer, R. Pieters, and W. Oosterlinck, Etiology of urethral stricture disease in the 21st century. J Urol, 2009, 182 (3): 983-7.

[4] Talreja, V., A. Ali, S. Saeed, K. Rani, S. S. Samnani, F. N. Farid, and M. Haider, Trail without Catheter after Transurethral Resection of Prostate: Clamp It or Not? Scientifica (Cairo), 2016, 2016: 1562153 .

[5] Durrani, S. N., S. Khan, and A. Ur Rehman, Transurethral resection of prostate: early versus delayed removal of catheter. J Ayub Med Coll Abbottabad, 2014, 26 (1): 38-41.

[6] Perera, N. D. and A. C. Nandasena, Early catheter removal after transurethral resection of the prostate. Ceylon Med J, 2002, 47 (1): $11-2$

[7] Walker, E. M., S. Bera, and M. Faiz, Does catheter traction reduce post-transurethral resection of the prostate blood loss? Br J Urol, 1995, 75 (5): 614-7.

[8] Sirivanasandha, B., P. H. Lennox, and H. Vaghadia, Transurethral resection of the prostate (TURP) with low dose spinal anesthesia in outpatients: a 5 year review. Can J Urol, 2011, 18 (3): 5705-9.

[9] Shih, H. J., Y. C. Chow, C. J. Huang, Y. H. Su, W. C. Lin, and S. Yang, Catheter-assisted transurethral resection of the prostate: a novel approach. Urol Int, 2008, 80 (4): 383-8.

[10] Teodorovich, O. V., N. B. Zabrodina, and A. B. Bochkarev, [Using setegis (terasosine) in early postoperative period after transurethral prostate resection]. Urologiia, 2009 (3): 62-4.

[11] Galante, D., An unusual cause of seizures during subarachnoid anesthesia in a patient undergoing transurethral resection of the prostate: a case report. Minerva Anestesiol, 2009, 75 (4): 221-3.

[12] Kim, N. Y., S. Y. Kim, H. M. Ju, and H. K. Kil, Selective spinal anesthesia using $1 \mathrm{mg}$ of bupivacaine with opioid in elderly patients for transurethral resection of prostate. Yonsei Med J, 2015, 56 (2): 535-42.

[13] Bhattacharya, B., A. Maung, K. Schuster, and K. A. Davis, The older they are the harder they fall: Injury patterns and outcomes by age after ground level falls. Injury, 2016, 47 (9): 1955-9.

[14] Joseph, B., V. Pandit, M. Khalil, N. Kulvatunyou, B. Zangbar, R. S. Friese, M. J. Mohler, M. J. Fain, and P. Rhee, Managing older adults with ground-level falls admitted to a trauma service: the effect of frailty. J Am Geriatr Soc, 2015, 63 (4): 745-9. 